\title{
In-Situ Focused Ion Beam Micropatterning of Ge Islands
}

\author{
M. Kammler*, R. Hull*, A. Portavoce*, M. C. Reuter**, and F. M. Ross** \\ *IBM T. J. Watson Research Center, Yorktown Heights, NY 10598 \\ **Depart. of Materials Science and Engineering, University of Virginia, Charlottesville, VA 22903
}

Self-assembly of islands during strained layer epitaxy is an established and successful method to form nanoscale structures which are exploit for their novel electronic and optical properties. Beyond the random nature of self-assembly being able to choose the island nucleation site will open future device applications such as quantum cellular automata [1].

The growth on Mesa structures or windows in an ultra thin oxide are successful methods to control nucleation sites [2,3], but with major disadvantages like not being able to incorporate the islands in an epitaxial silicon film. The ability to laterally pattern substrates without changing surface topography is therefore a key to novel device technologies. Addressing this problem, we found that focused ion beam (FIB) patterning is a promising tool to control the nucleation sites of Ge islands on $\mathrm{Si}(001)$ on a nanoscale [4].

The experiments were performed in our ultra high vacuum transmission electron microscope (UHVTEM) with the possibility to study, in real time, the growth process of thin films and epitaxial islands during chemical vapor deposition carried out in the microscope polepiece. Attached to the microscope is an additional UHV chamber housing a FIB column for sample patterning. After irradiating the specimen for $0.1 \mathrm{~ms} / \mathrm{spot}$ with a $25 \mathrm{keV} \mathrm{Ga}+$ ion beam (10pA beam current) we observe that each irradiated spot has intensity in the TEM surrounded by a ring of even higher intensity (Fig. 1 and Fig. 2a). Diffraction pattern indicate that amorphous material is present in the irradiated spots and that some amorphous material is also re-deposited in-between. We believe that direct implantation damage causes the central intensity, while re-deposited material from the sputter process causes the outer ring.

After FIB irradiation at RT we subsequently heat the patterned sample above $600^{\circ} \mathrm{C}$. Over a time frame of a few minutes the damage from the Ga irradiation anneals. Figure 2 illustrates the annealing process showing that the irradiated area anneals mainly from the outside forming a sharp ring of defects (Fig. 2b). With further annealing the circular contrast in the TEM image vanishes, leaving a few bright pinpoints below $6 \mathrm{~nm}$ in size (Fig. 2c). These spots may either be defect clusters or ultrasmall dislocation loops left after annealing or small $\gamma$-Ga precipitates. Using ex situ atomic force microscopy no change in topography can be observed after the annealing process, compared to the clean Si surface. We can actually see surface steps running continuously across the irradiated areas. Subsequently to the annealing process $\mathrm{Ge}$ island growth was performed at $600^{\circ} \mathrm{C}$ by introducing digermane into the sample area. After wetting layer growth, further Ge deposition leads to spontaneous island nucleation. The islands grow selectively at Ga-irradiated regions with no islands in-between. Recording the TEM contrast we observe that Ge islands below $20 \mathrm{~nm}$ in diameter are dislocation free while larger islands contain already dislocations. This is in strong contrast to the growth of Ge islands on clean $\mathrm{Si}(100)$ where no dislocations are observed in islands below 80nm in diameter. We suggest that the implanted Ga segregates to the surface during the annealing process acting as a surfactant changing the surface free energy and therefore the aspect ratio and strain energy of the island providing a low barrier for dislocation nucleation in the island. 
[1] C. S. Lent, et al., Nanotechnol. 4, 49 (1993).

[2] T. I. Kamins and R. S. Williams, Appl. Phys. Lett. 71, 1201 (1997).

[3] M. Ichikawa, J. Phys.: Condens. Matter 11, 9861 (1999).

[4] M. Kammler, et al., Appl. Phys. Lett. 82, 1093 (2003).
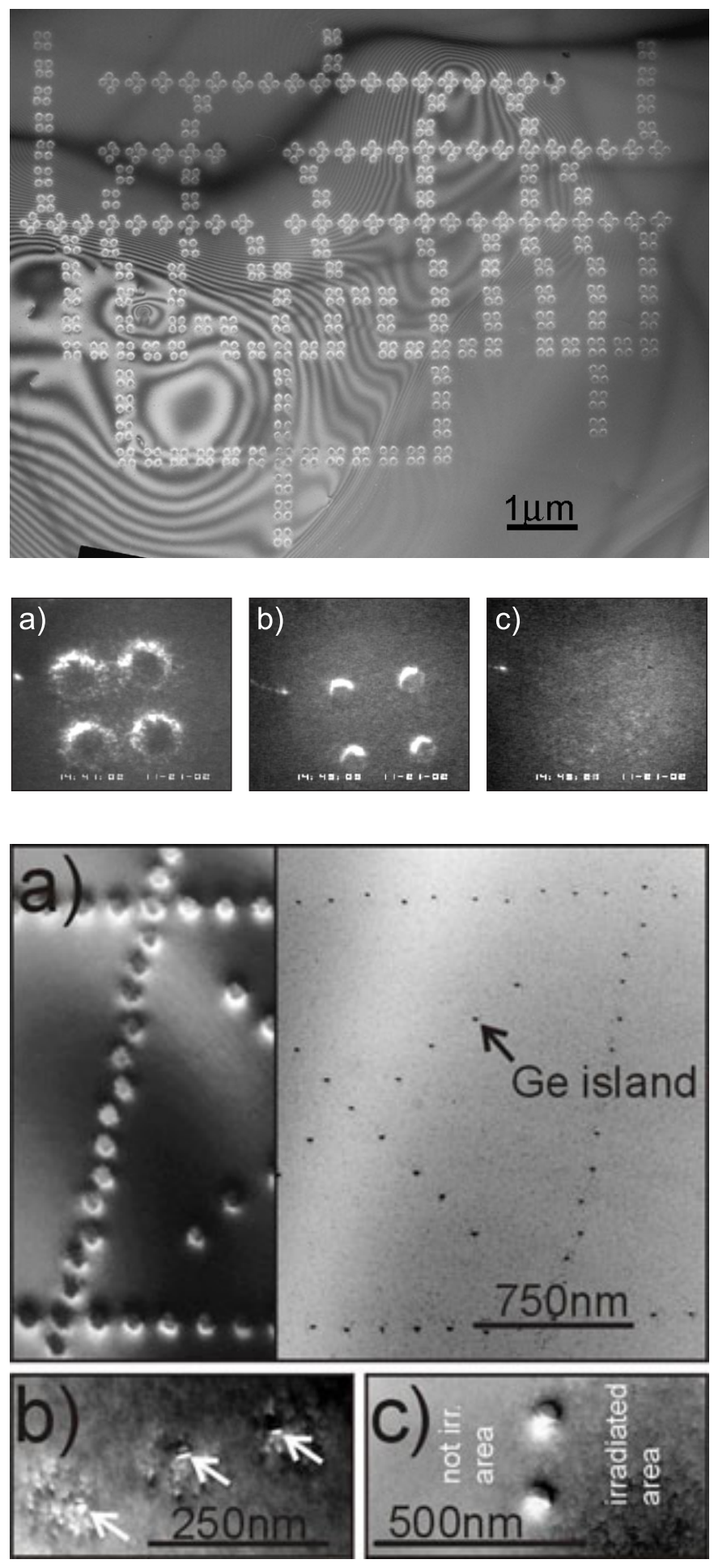

Figure 1: QCA adder pattern written with the FIB using a $25 \mathrm{keV} \mathrm{Ga}+$ ion beam $(10 \mathrm{pA})$ and an irradiation time of $0.1 \mathrm{msec} / \mathrm{spot}(6000 \mathrm{Ga}$ ions per spot). The planview TEM image is recorded using a $(\mathrm{g}, 3 \mathrm{~g})$ weak beam condition ( $g=220)$ sensitive to strained or damaged regions of the Si lattice.

Figure 2: Heating a cell of the QCA pattern slowly up to $600^{\circ} \mathrm{C}$. The TEM movie is recorded using a ( $\mathrm{g}, 3 \mathrm{~g}$ ) weak beam condition. (a) Before annealing. (b) During annealing. (c) After annealing.

Figure 3: (a) FIB pattern created using an irradiation time of $0.1 \mathrm{~ms}$, as-formed (left) and after annealing and Ge island growth (right). (b) Detail of irradiated areas after Ge growth. The arrows indicate single dislocation free $8.5 \mathrm{~nm}$ high Ge islands, only $20 \mathrm{~nm}$ in diameter, formed within each irradiated area. (c) Islands larger than $20 \mathrm{~nm}$ are already dislocated. 